\title{
Glycans as receptors for influenza pathogenesis
}

\author{
Karthik Viswanathan • Aarthi Chandrasekaran • \\ Aravind Srinivasan • Rahul Raman • V. Sasisekharan • \\ Ram Sasisekharan
}

Received: 10 June 2008 /Revised: 15 October 2008 /Accepted: 18 July 2010 /Published online: 24 August 2010

(C) Springer Science+Business Media, LLC 2010

\begin{abstract}
Influenza A viruses, members of the Orthomyxoviridae family, are responsible for annual seasonal influenza epidemics and occasional global pandemics. The binding of viral coat glycoprotein hemagglutinin (HA) to sialylated glycan receptors on host epithelial cells is the critical initial step in the infection and transmission of these viruses. Scientists believe that a switch in the binding specificity of HA from Neu5Ac $\alpha 2-3 \mathrm{Gal}$ linked $(\alpha 2-3)$ to Neu5Ac $\alpha 2-6$ Gal linked $(\alpha 2-6)$ glycans is essential for the crossover of the viruses from avian to human hosts. However, studies have shown that the classification of glycan binding preference of HA based on sialic acid linkage alone is insufficient to establish a correlation between receptor specificity of $\mathrm{HA}$ and the efficient transmission of influenza A viruses. A recent study reported extensive diversity in the structure and composition of $\alpha 2-6$ glycans (which goes beyond the sialic acid linkage) in human upper respiratory epithelia and identified different glycan structural topologies. Biochemical examination of the multivalent HA binding to these diverse sialylated
\end{abstract}

\footnotetext{
K. Viswanathan - A. Chandrasekaran · A. Srinivasan · R. Raman •

R. Sasisekharan $(\bowtie)$

Department of Biological Engineering,

Massachusetts Institute of Technology,

Cambridge, MA 02139, USA

e-mail: rams@mit.edu

V. Sasisekharan $\cdot$ R. Sasisekharan

Harvard-MIT Division of Health Sciences and Technology,

Massachusetts Institute of Technology,

Cambridge, MA 02139, USA

R. Sasisekharan

Koch Institute of Cancer Research,

Massachusetts Institute of Technology,

Cambridge, MA 02139, USA
}

glycan structures also demonstrated that high affinity binding of HA to $\alpha 2-6$ glycans with a characteristic umbrella-like structural topology is critical for efficient human adaptation and human-human transmission of influenza A viruses. This review summarizes studies which suggest a new paradigm for understanding the role of the structure of sialylated glycan receptors in influenza virus pathogenesis.

Keywords Hemagglutinin · Multivalency · Sialylated glyans · Glycan binding preference

\section{Introduction}

Complex polysaccharides or glycans comprise a major part of the cell surface and extra-cellular matrix of epithelial cells [1]. Cell surface glycans, which are covalently attached to proteins to form glycoconjugates, are highly heterogeneous and can be linear or branched in structure. Defining the structure-function relationships of these glycans enabled the understanding of their diverse biological roles $[2,3]$. Glycans play a role in several physiological and pathological processes, such as cell growth and differentiation; cell-cell and cell-matrix signaling; cell adhesion and tumor invasion; and metastasis; through their interactions with growth factors, enzymes, and other ligands $[1,2]$. Studies have also indicated an important role for these complex polysaccharides in microbial pathogenesis and invasion. Several microbial pathogens utilize glycans at the host cell surface and extracellular environment as attachment sites or receptors that enhance their ability to infect and invade host epithelia [4]. The binding of pathogens to glycan receptors on host cells is permitted by microbial surface proteins possessing specific 
carbohydrate recognition domains. Influenza A virus is a pathogen that attaches to terminal sialic acid glycan receptors on the host epithelial cell surface [5]. For several decades scientists have known that the binding of the influenza viral coat protein hemagglutinin (HA) to sialylated glycans on the host cell surface is a key first step for viral entry and infection [6]. Over the years however, perception of the role that sialylated glycan receptors play in this infection process has altered considerably. Advancement of analytical techniques to characterize physiological glycans, improved availability of co-crystal structures of influenza hemagglutinin with sialylated glycan receptors [7] and development of glycan arrays to study protein-glycan interaction [8] have all significantly enhanced our understanding of the role of sialylated glycans in influenza A virus pathogenesis.

\section{Influenza viruses}

Influenza A viruses are a major cause of flu epidemics annually. In a typical year approximately $15 \%$ of the U.S. population is infected, resulting in about 36,000 deaths and more than 200,000 hospitalizations (www.cdc.gov/flu). These viruses occasionally mutate to become highly pathogenic - which could lead to a widespread pandemic and serious global health concerns. Based on the estimated mortality rate of the 1918 Spanish flu pandemic, epidemiological studies predict that a modern pandemic would affect as many as 75 million people worldwide.

Influenza A viruses are negative-sense, single-stranded RNA viruses of the Orthomyxoviridae family [9]. These viruses exist in a variety of shapes: ranging from spherical particles to elongated filamentous forms, and sizes ranging from 80-120 nm. Their genome consists of eight RNA segments encodes for eleven viral proteins [9-12]. Three of these proteins: hemagglutinin (HA), neuraminidase (NA), and matrix protein (M1) are present on the surface of influenza viruses. Other encoded proteins include RNA polymerase subunits (PB1, PB2, PA), nucleoprotein (NP), nonstructural proteins (NS1 and NS2), a proton-selective ion channel protein (M2) and a newly discovered proapoptotic PB1-F2 protein [13]. Influenza viruses lack a proofreading mechanism in their RNA polymerase, which causes the synthesis of new transcripts to be error prone. Accumulations of these mutations permit the development of new serotypes in a process known as antigenic drift. This antigenic drift helps the virus evade its' host immune system by significantly refashioning the antigen present on the surface. Transmembrane proteins HA and NA are the antigenic determinants of these viruses and form the basis for their classification into different serotypes. $16 \mathrm{HA}$ and 9 NA serotypes have been identified thus far. The segmented nature of viral RNA allows reassortment of genes between viruses of different serotypes and results in new subtypes. This process is called antigenic shift. Both antigenic drift and antigenic shift are part of the evolution of an influenza virus [14]. Antigenic drift is a continuous process and leads to gradual changes in surface antigens while antigenic shift is an occasional occurrence and leads to significant changes in the antigen present on the virus surface.

Influenza viruses infect numerous animal species. Wild aquatic birds serve as a natural reservoir for these viruses. They also intermittently spread to other species such as domestic birds, swine, and humans [15-18]. These viruses are responsible for the seasonal flu epidemics and more sporadic pandemics in humans. Two specific subtypes (H1N1 and H3N2) of these viruses currently circulate in humans and are the cause for seasonal flu epidemics. Influenza A viruses occasionally evolve into highly pathogenic and virulent strains either by acquiring mutations or entire gene segments through reassortment in a host infected by more than one viral strain. Highly pathogenic strains yield a high mortality rate in humans and efficiently transmit from human-to-human, which results in widespread pandemic and serious global health concerns [10, 11, 19, 20]. Among different strains of avian influenza viruses such as H2N2, H5N1, H7N7 and H9N1, which are currently known to infect humans, the H5N1 or "bird flu" virus is by far the deadliest (with a mortality rate greater than $60 \%$, notably higher than pandemic strains) [21-25]. Fortunately, these viruses have not yet acquired the ability to efficiently transmit between humans $[26,27]$. Outbreaks have therefore been localized to specific parts of the world. The H5N1 strains and others have the potential to become serious global threats if they mutate to adapt to human hosts and achieve efficient human-human transmission.

The virus infection cycle of influenza A begins with the attachment of the virus to glycan receptors on the host cells. The virus is then internalized by endocytosis, followed by membrane fusion and virus uncoating. Subsequently, the viral RNA is transported to the nucleus where it undergoes replication and transcription. Newly synthesized proteins HA and NA are secreted through the Golgi to the cell surface, while other proteins are transported to the nucleus to associate with the synthesized transcripts of viral RNA and form virions. These virions then bulge out of the cell membrane with HA and NA at the surface. The action of neuraminidase facilitates the release of these progeny viruses from their host cells that go on to infect other cells.

Influenza viruses are well known for their ability to agglutinate red blood cells (RBCs) in vitro. HA, which is responsible for the agglutination, is a transmembrane glycoprotein that exists as a homotrimer with the molecular 
weight of $220 \mathrm{kDa}$ [28]. HA is a type 1 integral membrane protein with a N-terminal hydrophobic signal sequence that is cleaved off once the protein is anchored to the membrane, a transmembrane anchoring domain near its Cterminal, and a short hydrophilic cytoplasmic tail [7]. HA plays an important role in different stages of viral pathogenesis. As noted earlier, HA functions on the viral surface as a glycan binding protein and enables viral attachment to host epithelia by binding to sialylated glycan receptors on the host cell surface. In subsequent steps of viral pathogenesis, HA also undergoes a $\mathrm{pH}$ dependant conformational change and aids in the fusion of viral and host endosomal membranes post-endocytosis $[6,7]$.

\section{Human glycan receptors for influenza virus infection}

The ability of the virus to agglutinate chicken red blood cells was first observed by Hirst et al. in 1941 [29], but it was only in the 1950s that the identity of the receptor on these erythrocytes was discovered. Pretreatment of erythrocytes with culture filtrates of Vibrio cholerae destroyed the ability of these erythrocytes to agglutinate in the presence of influenza viruses [30]. The split product of the enzyme treatment was subsequently shown to be sialic acid [31-33]. Sialic acids are a diverse family of 9-carbon monosaccharides, of which the most abundant member is $\mathrm{N}$-acetylneuraminic acid. They are negatively charged and present either at the terminal position of the glycan or linked to another sialic acid [34]. Sialic acid is added on to $\mathrm{N}$ - or $\mathrm{O}$-glycans by the sialyltransferase family of enzymes. The sialyltransferase responsible for the addition of sialic acid depends on the monosaccharide it is added to and the linkage it is attached with. Sialic acid is linked to the penultimate sugar - usually a galactose (Gal) - in either $\alpha 2-3$ linkage or $\alpha 2-6$ linkage. When sialic acid is added to another sialic acid, it attaches with an $\alpha 2-8$ linkage.

Due to its terminal position, it is this monosaccharide that is most likely to interact with extracellular molecules including pathogens [35]. The human upper respiratory tract is the primary site of influenza A virus infection and the glycans on the epithelial cell surface that are human receptors for these viruses are capped with sialic acid. Early studies with lectin staining revealed that human tracheal and bronchial epithelia predominantly express $\alpha 2-6$ sialylated glycans [36]. Shinya et al. recently demonstrated the extensive presence of $\alpha 2-6$ sialylated glycans in nasal mucosa, on epithelial cells of paranasal sinuses and the pharynx, and presence in the trachea and bronchi [37]. Human deep lung alveolar cells are, on the other hand, lined with $\alpha 2-3$ sialylated glycans. It is important to note that $\alpha 2-3$ sialylated glycans are extensively expressed at the site of infection in birds [38].

\section{Glycan receptor binding specificity of influenza virus HA}

Molecular HA-glycan interactions: crystal structure analysis

The HA crystal structure first resolved was the HA from the H3N2 strain: A/Hong Kong/1968 by Wilson et al. [28]. This trimeric HA unit is cylindrical with a length of $135 \mathrm{~A}$ and radius varying between 15 to $40 \AA$. It has a globular head region that comprises the glycan binding site. Structures of several HA proteins from $\mathrm{H} 1, \mathrm{H} 3, \mathrm{H} 5, \mathrm{H} 7$ and $\mathrm{H} 9$ subtypes have also been solved, along with the co-crystal structures of HA with $\alpha 2-3$ and/or $\alpha 2-6$ sialylated oligosaccharides [3945]. Analysis of these HA-glycan co-crystal structures indicated that amino acids are involved in making contact with the sialylated glycan. In each case, the position of Neu5Ac sugar was found to be fixed relative to the HA glycan binding site, with a highly conserved set of amino acids: Tyr98, Ser/Thr136, Trp153, Thr155, His183, Leu/ Ile194 (amino acid numbering is based on H3 HA) across different HA subtypes anchor the Neu5Ac [6]. The linkage of Neu5Ac to the galactose $(\mathrm{Gal})$ residue determines the cis- or trans- conformation adopted by the glycan in the HA-glycan co-crystal structures. In the HA- $\alpha 2-3$ co-crystal structures, Neu5Ac $\alpha 2-3 \mathrm{Gal}(\alpha 2-3$ motif) adopts a trans conformation. In this conformation, in addition to the conserved anchor points for sialic acid binding two critical residues of HA - Glu190 and Gln226 - are involved in binding to the $\alpha 2-3$ motif. Located at the base of the binding site, Gln226 interacts with the glycosidic oxygen atom of the Neu5Ac $\alpha 2-3 \mathrm{Gal}$ linkage; and, on the opposite side of Gln266, Glu190, interacts with Neu5Ac and Gal monosaccharides [40, 41, 46]. In the HA$\alpha 2-6$ co-crystal structures, Neu5Ac $\alpha 2-6 \mathrm{Gal}$ ( $\alpha 2-6$ motif) contrarily adopts a cis conformation. In this conformation, the critical amino acids of HA involved in stabilizing the glycan interaction are subtype specific, but conserved within a subtype. In H1 HAs, Lys222 and Asp225 are positioned to interact with the oxygen atoms of the Gal in the $\alpha 2-6$ motif and Asp190 and Ser/Asn193 interact with additional monosaccharides GlcNAc $\beta 1-3 \mathrm{Gal}$ linked to

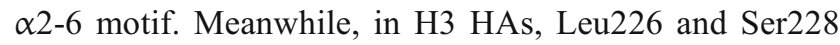
are positioned to interact with the $\alpha 2-6$ motif.

Experimental methods to characterize HA-glycan interactions

Numerous methods have been developed to biochemically characterize HA-glycan interactions. The earliest method developed to probe the glycan specificity of HA was a study of its ability to agglutinate red blood cells (RBCs). RBCs from species such as chicken, turkey, equine, guinea pigs, and humans have been used for these agglutination assays [47-51]. The predominance of $\alpha 2-3$ on equine RBCs and $\alpha 2-6$ on turkey and guinea pigs RBCs made 
them appropriate tools to probe HA specificity. RBCs from almost all species possess both $\alpha 2-3$ and $\alpha 2-6$, so this agglutination method was further modified to include a step of complete desialylation of the RBCs, followed by sialylation by specific $\alpha 2-3-$ and $\alpha 2-6-$ sialyltransferase, to make the agglutination assay more reliable when probing sialic acid linkage specificity. Subsequent development of solid-phase fetuin capture assays offered a wealth of information on the glycan binding properties of influenza A viruses. In these assays, the viruses are immobilized on fetuin-coated surfaces and their binding to various sialylated glycans (including polyvalent compounds) is then evaluated [16, 52-55]. The advent of the chemoenzymatic synthesis of glycans and development of the glycan array platform permitted study of the HA specificity to chemically defined glycans [56-58]. Glycan arrays developed by the Consortium of Functional Glycomics (www. functionalglycomics.org) comprise several $\alpha 2-3$ and $\alpha 2-6$ oligosaccharides, which cover motifs corresponding to both O-linked and N-linked glycans [8, 59, 60]. Intact viruses, recombinantly expressed HAs, and their mutant forms (glycan-binding variants) from $\mathrm{H} 1, \mathrm{H} 3$, and $\mathrm{H} 5$ subtypes have already been analyzed using these glycan arrays $[8,44,61]$. These studies have classified binding preference based on the number of distinct $\alpha 2-3$ and $\alpha 2-6$ glycans that possess detectable binding signals for a given HA. These studies also provide an understanding of the effects of substitutions such as sulfation and fucosylation and sugar modifications on the HA-glycan interactions.

\section{HA-glycan interactions: receptor binding specificity}

Inspection of the different influenza viruses using the above-mentioned techniques reveals that HAs from different species differ in their receptor specificity. This was first demonstrated by Rogers and Paulson in 1983 using $\alpha 2-3$ or $\alpha 2-6$ resialylated RBCs [48]. Most avian influenza viruses predominantly bind to sialic acid in $\alpha 2-3$ linkage, whereas the HAs from human adapted viruses predominantly recognize sialic acid which is $\alpha 2-6$ linked to the underlying sugar. Most studies focused on characterizing virus specificity based on sialic acid linkage only, but occasional studies instead probed the influence of the internal sugars on the viruses' receptor specificity. Studies by Gambaryan et al. showed the differences in the binding ability of human adapted HAs to Neu5Ac $\alpha 2-6 \mathrm{Gal} \beta 1-4 \mathrm{GlcNAc}$ and Neu5Ac $\alpha 2-6 \mathrm{Gal} \beta 1-4 \mathrm{Glc}[62]$. Avian viruses isolated from different species similarly show a common preference to $\alpha 2-3$ sialylated glycans but differ in their recognition for glycan modification beyond the Gal residue [53]. In spite of these results, the sialic acid linkage was believed to be the key determinant of receptor specificity and crossover of the viruses from birds to humans was associated with the ability of HA to switch its receptor specificity from $\alpha 2-3$ sialylated glycans to $\alpha 2-6$ sialylated glycans.

HA-glycan binding: role of receptor specificity in viral transmission

The ability to completely reconstruct the pandemic 1918 H1N1 viruses through reverse genetics predicated breakthroughs in the exploration of the roles of the different viral genes - especially HA - and their virulence and transmissibility $[11,63]$. The relationship between the HA glycan binding specificity and transmission efficiency was demonstrated recently in a ferret model using the 1918 H1N1 viruses. Ferrets offer reliable animal models to study human transmission of influenza viruses due to the predominance of human-like $\alpha 2-6$ glycans in their upper respiratory tract epithelium [26]. In the Tumpey et al. study a prototypic 1918 pandemic virus SC18 and two other variants were used. One variant was created by mutating the Asp225 to Gly225 on the HA to reflect natural variant A/New York/1/ 18 (NY18) isolated from an archived lung sample. The second variant (AV18) was created by introducing an additional mutation (Asp190 to Glu190) on NY18 HA to match the conserved HA sequence of avian H1N1 viruses. SC18, NY18 and AV18 viruses differ only in their HA sequence while all other 7 genes of the virus are identical. The mutations in HA resulted in differences in their glycan receptor binding preference. SC18 virus demonstrated a preference for $\alpha 2-6$ sialylated glycans; NY18 showed binding to both $\alpha 2-3$ and $\alpha 2-6$ sialylated glycans and AV18 bound only to $\alpha 2-3$ sialylated glycans. In ferrets, only the SC18 virus displayed efficient respiratory droplet transmission. NY18 transmitted inefficiently and AV18 did not transmit. These findings suggested that a switch in viruses' HA receptor specificity from $\alpha 2-3$ to $\alpha 2-6$ sialylated glycans could be imperative for efficient transmission of these viruses. However in this same study, a contemporary H1N1 strain, A/Texas/36/91 (Tx91) that had a mixed $\alpha 2-3 / \alpha 2-6$ binding was able to transmit efficiently [50]. Observed differences in the transmissibility of NY18 and Tx91 despite their both having mixed $\alpha 2-3 / \alpha 2-6$ sialylated glycans binding indicated that the "switch" framework does not adequately explain the role of receptor specificity of HA in human adaptation and transmission of influenza A viruses. This observation requires the detailed understanding of HA-glycan interaction, beyond their binding to sialylated glycans with a specific linkage, and the role of such interactions in virus transmission.

HA-glycan binding: sialic acid linkage and beyond

Agglutination and other traditional hemagglutination assays enable the determination of viruses' specificity 
according to the sialic acid linkage it binds to. However a major drawback still remains in the failure to permit the examination of specificity beyond this sialic acid linkage per se. Other traditional methods, such as fetuin capture assays $[64,65]$ offer the same limitations due to the limited set of $\alpha 2-3$ and $\alpha 2-6$ sialylated oligosaccharides used in these assays. This characterization of the glycan binding specificity of influenza virus HA in terms of $\alpha 2-3$ and $\alpha 2-6$ sialic acid linkage alone does not adequately capture the substantial diversity, chemical heterogeneity and conformational flexibility of sialylated glycans or the functional roles of the HA-glycan interactions.

The interaction of sialylated glycans with HA, as with other glycan-protein interactions, is multivalent and involves an ensemble of glycans making contact with the glycan-binding site of the protein. Although the glycan binding affinity for a single binding site on any glycan binding protein (such as a lectin) is typically low (in the high $\mu \mathrm{M}$ to $\mathrm{mM}$ range), it is compensated by the avidity effects of the multivalent interaction. Multivalency further complicates the study of the biochemical basis of HA-glycan interactions [66]. Assays developed for studying HA-glycan interactions therefore need to capture this multivalency.

The development of glycan array platforms has dramatically expanded the diversity of glycans employed to investigate HAglycan interactions [8]. The presentation of glycans on the array partly mimics the physiological presentation of glycans on the cell surface. Glycan array binding of whole viruses ensures the appropriate presentation of the viral HA. To enhance the multivalent presentation of recombinantly expressed HA proteins (partly mimicking the viral HA presentation), methods have been developed to precomplex the HA with labeled antibodies and detecting the binding signal of the entire precomplexed units on the glycan array [8]. Despite these developments, the major limitation in the interpretation of the glycan array data arises from the assays being performed with a single concentration of HA, or at a single viral titer [8]. Analysis of the binding of HA to sialylated glycans at a single HA concentration - especially in saturating conditions-precludes observation of the finer nuances of glycan specificity. Analysis at multiple concentrations - or viral titers - in the dynamic range of the assay system would enable improved quantification of binding affinity parameters.

These experimental challenges necessitate the development of integrated approaches to permit study of complex HA-glycan interactions [67]. A set of complementary approaches would entail (1) an investigation of the diversity of the sialic acid structures present in the human upper airways, (2) a detailed analysis of the three dimensional structure of the sialylated glycans predominantly expressed in human upper airway epithelia, (3) a molecular understanding of HA-glycan interaction beyond the sialic acid linkages, and (4) a thorough biochemical characterization of HA-glycan binding specificities, which takes into account the multivalent nature of these interactions [67].

When employing the above complementary approaches, the first step is an analysis of the physiological glycan receptors present in the upper airway and further characterization of its structural attributes beyond simple sialic acid linkage. The analysis of glycans directly from the surface of human epithelia is confounded by challenges to their accessibility. Glycan binding proteins such as plant lectins are widely used to characterize glycans present on human tissues. Although early studies with lectins such as Sambucus nigra agglutinin (SNA-I) revealed the predominance of $\alpha 2-6$ sialylated glycans on the epithelial surface of human tracheal sections [68], recently a wider panel of lectins has been used to elaborate the diversity of glycans beyond the sialic acid linkages [67]. Lectin co-staining experiments indentified a widespread distribution of $N$ linked $\alpha 2-6$ sialylated glycans on the ciliated cells in contrast with the localized distribution of $O$-linked $\alpha 2-6$ sialylated glycans on the goblet cells on the apical side of the tracheal section [67]. A limitation in the availability of glycans extracted form the tissues precludes their further characterization. To gain further insight in the diversity of glycan structures present, a representative upper airway cell line was selected and glycans on their surface characterized using MALDI-MS and MS-MS analytical techniques. MALDI-MS profiling of $N$-linked sialylated glycans isolated from this representative human bronchial epithelial (HBE) cell line showed the expected predominance of $\alpha 2$ 6 sialylated glycans. Moreover, substantial diversity was found in the length and branching of the $\alpha 2-6$ sialylated glycans present [67]. Further analysis of the major glycan species present using MS/MS profiling revealed the presence of long oligosaccharide branches with multiple lactosamine repeats [67].

The diversity of $\alpha 2-6$ sialylated glycans present in the upper airway prompted re-examination of the available HA-glycan co-crystal structures and analyses of the conformational features of these glycans, taking into account additional parameters such as length and branching. The Neu5Ac (SA) sugar is fixed relative to the HA glycan binding site in all the co-crystal structures. These investigations also showed that the additional sugars beyond the Neu5Ac adopt two distinct shapes or threedimensional topologies which provide optimal contacts with the different HAs (Fig. 1). In one of the topologies, the sugars underlying the SA (such as Gal, GlcNAc, etc.) span a cone-like region on the HA glycan binding surface and hence is known as cone-like topology. This topology was typically adopted by $\alpha 2-3$ sialylated glycans, short (di/tri-saccharide) $\alpha 2-6$ oligosaccharide motifs such as 6 '-sialyl-lactose (or lactosamine) (Neu5Ac $\alpha 2-6 \mathrm{Gal} \beta 1$ - 
4Glc(NAc) $\beta 1$-), and 6'-sialyl-Tn- antigen (Neu5Ac $\alpha 2$ 6 GalNAc $\alpha 1-)$. Another topology, identified as umbrellalike was such that at least three sugars in addition to the Neu5Ac spanned a wider region on the HA glycan binding surface (as compared to the cone-like topology) that resembled a space that could be defined by anywhere between a fully-closed to a fully-opened umbrella. The cone-like topology that could be adopted by sialylated glycans having both $\alpha 2-3$ and $\alpha 2-6$ linkages but the umbrella-like topology was unique to longer $\alpha 2-6$ oligosaccharide motifs such as $6^{\prime}$-sialyl-polylactosamine (Neu5Ac $\alpha 2-6[\mathrm{Gal} \beta 1-4 \mathrm{GlcNAc} \beta 1-3]_{2}-$ ). The classification of binding of HA to a particular glycan topology adequately captures the effect of glycan structural diversity on the specificity of HA-glycan interactions in contrast to a classification based on HA binding to a glycan of a specific $\alpha 2-3$ or $\alpha 2-6$ linkage in the trans or cis conformations, respectively [67, 69].

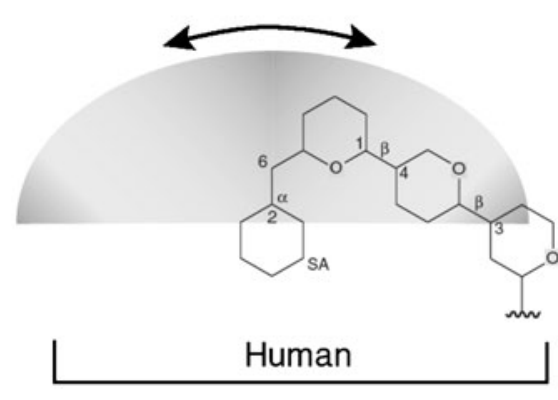

Umbrella-like

Conformational sampling of $\alpha 2-3$ linkage

\begin{tabular}{|l|c|}
\hline Cone-like & $100 \%$ \\
\hline Umbrella-like & $0 \%$ \\
\hline
\end{tabular}

HA interactions with Cone Topology

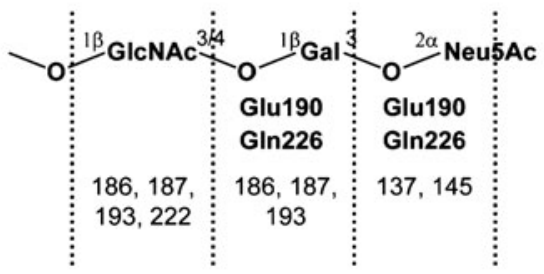

Fig. 1 Structural topology of sialylated glycans. Shown at the top is a schematic view of the umbrella-like and cone-like topologies that respectively distinguish the glycan-receptors for the human-adapted and the avian (and swine) influenza A viruses. This part of the figure was adapted from Bewley, C.A. (2008) Nature Biotechnol. [69]. Shown in the middle is the propensity of the $\alpha 2-3$ and $\alpha 2-6$ sialylated glycans to adopt the distinct cone-like and umbrella-like topologies. The conformational energy maps of Neu5Ac $\alpha 2-3 \mathrm{Gal}$, Neu5Ac $\alpha 2-$ $6 \mathrm{Gal}$ and Gal $\beta 1-4 \mathrm{GlcNAc}$ linkages in a trisaccharide (Neu5Ac $\alpha 2-3$ / 6Galß1-4GlcNAc-) motif were obtained from GlycoMaps DB (http:// www.glycosciences.de/modeling/glycomapsdb/). The glycan topology was characterized using a parameter $\theta$ (angle between $\mathrm{C} 2$ atom of

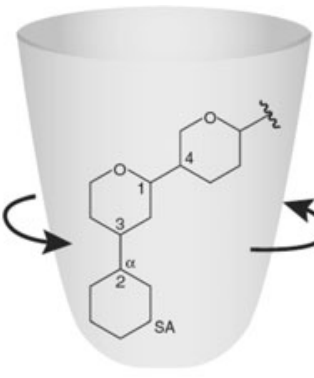

Avian

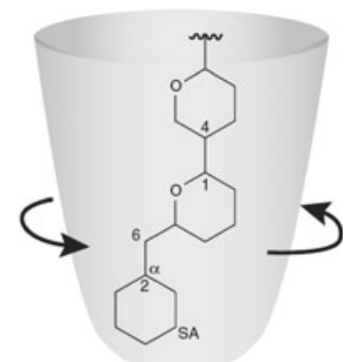

Avian, swine
Cone-like

Conformational sampling of $\alpha 2-6$ linkage

\begin{tabular}{l|c} 
& Umbrella-like (\%) : Cone-like (\%) \\
\hline$\omega=-60^{\circ}$ & $60: 20$ \\
\hline$\omega=+60^{\circ}$ & $10: 40$ \\
\hline$\omega=180^{\circ}$ & $30: 30$ \\
\hline
\end{tabular}

\section{HA interactions with Umbrella Topology}

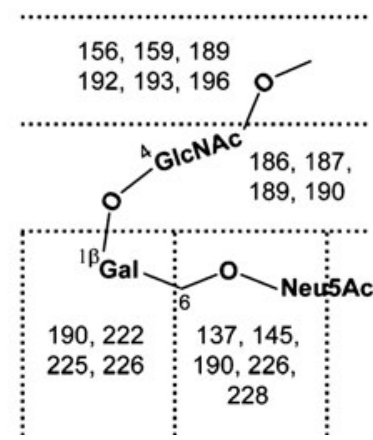

Neu5Ac and $\mathrm{C} 1$ atoms of subsequent Gal and GlcNAc sugars) for a given set of $(\varphi, \psi)$ angles between the linkages. The value of $\theta>110^{\circ}$ was used to characterize cone-like topology and $\theta<100^{\circ}$ was used to characterize umbrella-like topology. Superposition of the $\theta$ contour with the conformational energy map indicated that $\alpha 2-3$ motif adopts $100 \%$ cone-like topology since it was energetically unfavorable to adopt umbrella-like topology. On the other hand, the $\alpha 2-6$ motif sampled both the cone-like and umbrella-like topologies and this sampling was classified based on the $\omega$ angle (O-C6-C5-H5) of Neu5Ac $\alpha 2-6$ Gal linkage. Shown in the bottom are the key HA residue positions (numbered based on H3 HA) that interact with the specific sugars in the cone-like and umbrella-like topologies 
HAs from a number of H1N1, H3N2 and H5N1 strains and their mutants have been analyzed on the glycan array for binding to previously defined glycan structures [44, 70]. Data mining analysis of existing glycan array data led to a generation of classifiers or rules that correlate the binding of different HAs to the various structural and conformational features of the glycans on the array [67]. This analysis revealed that the common feature between the HAs from human adapted $\mathrm{H} 1 \mathrm{~N} 1$ and $\mathrm{H} 3 \mathrm{~N} 2$ viruses is their specific binding to the long $\alpha 2-6$ glycans that adopt an umbrella-like topology. Subsequent biochemical assays examined the binding specificities of the different HAs and validated the findings from these analyses. In this investigation HA expressed in insect cells was presented in a multivalent fashion to a previously determined representative set of glycans with different linkages and topologies. As anticipated, the results correlated with previous analyses: the HAs from human adapted $\mathrm{H} 1$ and $\mathrm{H} 3$ strains specifically bound to the longer $\alpha 2-6$ oligosaccharide (with multiple lactosamine repeats) which adopts the umbrella-like topology [67]. These findings indicate that binding to glycans with a distinct umbrella-like topology is critical for the human adaptation of HA from influenza A viruses. This study also highlights the finding that linkage alone cannot adequately explain the human adaptation and transmission of influenza A viruses. Glycan structure and topology are instead crucial considerations in any examination of receptor specificities of influenza viruses.

\section{Revisiting the 1918 pandemic H1N1 virus}

The above study redefined the criteria for the human adaptation and transmission of influenza viruses by accounting for the glycan topology and going beyond the sialic acid linkages. Could a framework that classifies HAglycan interaction based on glycan topology shed light on the differences in transmission of the different 1918 viruses and also explain the conundrum of NY18 and Tx91 transmission? To answer this question, these new concepts were applied to the 1918 H1N1 virus system. Based on the transmission efficiencies of the different 1918 viruses, one would expect that $\mathrm{SC} 18$ (which efficiently transmits in ferret models) would bind to umbrella-like topology glycans, while AV18 (with inefficient transmission) will show minimal binding to umbrella-like glycans. The analysis of binding of the mixed binding NY18 virus (which inefficiently transmits in ferrets) to glycans with different topologies would also provide useful insights for understanding the role of receptor binding specificities in viral transmission.

An in silico analysis of the HA-glycan co-crystal structures revealed differences in the HA interaction with glycans in cone-like and umbrella-like topology [71]. The crystal structure of SC18 HA has been resolved and the NY18 and AV18 structures were obtained in silico by building mutations on this known structure of SC18. Cocrystal model of SC18 with long $\alpha 2-6$ glycan in umbrellalike topology indicate that the HA makes contact with the glycan in two distinct regions. The Gal residue linked to sialic acid forms the base region of the $\alpha 2-6$ motif and additional monosaccharides GlcNAc $\beta 1-3 \mathrm{Gal}$ form the extension region of the $\alpha 2-6$ motif. In AV18 mutations at positions 225 and 190 prevent it from making critical contacts to the base and extension regions. This renders it incapable of interaction with glycans in umbrella-like topology. AV18 instead interacts with glycans in cone-like topology. The absence of the critical Glu190 residue in SC18 and NY18 HAs makes their interaction with glycans in cone-like glycan topology less optimal in comparison with AV18 HA.

Differences in the binding specifities of SC18, NY18 and AV18 HAs to glycans in cone-like and umbrella-like topologies were captured in direct binding assays with plated glycans. To capture the multivalent nature of HAglycan interactions, SC18, NY18 and AV18 HAs were precomplexed with primary and secondary antibodies (HA: primary: secondary $=4: 2: 1$ ratio). This allowed exploration into the effects of the relative spatial positioning of multiple HA units on glycan binding affinity. By performing these assays in a dose dependent fashion, the relative glycan binding affinities of HAs with long and short $\alpha 2-6$ and $\alpha 2-$ 3 were also evaluated. Quantitative parameters such as the apparent binding constant $K_{d}^{\prime}$ and cooperativity factor $n$ were calculated by fitting binding data to a linearized Hill model. As anticipated from the analysis of co-crystal structures, SC18 HA showed high binding affinity to the long $\alpha 2-6$ sialylated glycan. Also as expected, AV18 HA showed minimal binding to $\alpha 2-6$ sialylated glycans and high binding affinity to $\alpha 2-3$ sialylated glycans. Interestingly, NY18 HA showed binding to umbrella-like topology glycans, but had a significantly lower binding affinity in comparison with SC18 for long $\alpha 2-6$ sialylated glycans. HAs from other representative human adapted viruses (such as H3N2) also displayed high binding affinities for umbrella-like topology glycans. These findings suggest that binding to umbrella-like topology glycans with high binding affinities is critical for efficient human to human transmission of the virus [71].

In a similar analysis, Tx91 HA was shown to have binding affinities comparable to those of $\mathrm{SC} 18$ for the umbrella topology glycan. The observed difference in the binding affinities of Tx91 and NY18 HA to long $\alpha 2-6$ or umbrella-like glycans could explain the observed difference in transmission of these viruses despite a shared mixed $\alpha 2-3 / \alpha 2-6$ binding [71]. 


\section{Physiological relevance of $\mathrm{HA}$ binding to glycans}

Despite the advantages that glycan array assays offer in terms of valuable information about the glycan specificity of HA, our understanding of HA-glycan interaction is incomplete without elucidating its physiological relevance. Glycan arrays possess various glycan motifs but still do not capture the diversity of glycans present on the cell surface. It is important to bridge the glycan specificity of HA on the array to its ability to bind to glycan receptors at the site of infection. Pre-complexed HA from different strains have been used in a manner similar to plant lectins, to stain tracheal tissue sections. Tracheal tissue contains multiple cell types including cartilage ring, pseudostratified ciliated epithelium, goblet cells and submucosal gland cells. From these, the ciliated cells and goblet cells are exposed on the surface and glycans on these cells are potential receptors for influenza viruses.

Notably, the HAs from human-adapted viruses (such as $\mathrm{SC} 18$ and two representative H3N2 viruses) examined in the preceding analyses bind to the apical side of the tracheal epithelium, which lectin and MS analysis show a predominant expression of glycans, which adopt the umbrella-like topology. Comparison of binding patterns of $\mathrm{SC} 18$ and NY18 HA to the apical side of the trachea reveals a more restricted binding of SC18 when compared to the welldistributed binding of NY18 [71]. This suggests that SC18 binds to more selective cell types than NY18. Observation of differences in the binding patterns of HAs from different viruses requires further investigation of the structural and conformational diversity of the $\mathrm{N}$-linked and O-linked sialylated glycans in these different cell types in the trachea. The diversity and distribution of sialylated glycans in each cell type could determine the cellular tropism of the different influenza A viruses. Additional study is needed to examine the correlation between these viral tropisms and differences in the efficiency of the transmission of influenza A viruses. Little is still known about the structure and composition of sialylated glycans in other human upper respiratory tissues such as nasopharangeal, bronchial and alveolar. A combination of lectin staining of these tissues and MS analysis of representative cell lines could provide valuable information on the composition and topologies of the glycan receptors expressed in the human respiratory tract.

\section{Implications for H5N1 and future work}

Avian H5N1 virus strains currently circulate worldwide and cause epidemics in poultry. Since 1997 occasional reports of these viruses infecting humans have emerged. These viruses have a very high mortality rate but have not yet achieved efficient human-human transmission. Glycan array analysis of these strains reveals their propensity to bind to $\alpha 2-3$ sialylated glycans $[44,70]$. Although some of these strains and some lab-created mutants have been shown to have binding to $\alpha 2$ 6 sialylated glycans, they still lack the ability to cause human-to-human transmission. This is because the $\alpha 2-6$ binding of these viruses are either limited to short $\alpha 2-6$ sialylated glycans that take the cone-like topology similar to $\alpha 2-3$ sialylated glycans or - even if the viruses bind to long $\alpha 2-6$ sialylated glycans - their binding affinity is low [67].

Performance of the assay in a dose dependent fashion to capture affinity and characterization of the ability of the virus to bind to glycans in umbrella-like topology would facilitate the determination of HAs' actual adaptation for human-to-human transmission as well as permit for effective surveillance of their evolution into potential pandemic human viruses. Further defining the determinant for human adaptation of HA allows for the rational design and experimental validation of HA mutations that would enable high affinity binding to the $\alpha 2-6$ glycan receptors with the characteristic umbrella-like topology. This knowledge would enhance our preparedness for any pandemic threat and facilitatethe development of prophylactic vaccines and possible glycomimetic therapeutics.

\section{Concluding remarks}

The attachment of HA on the influenza virus to the sialylated glycan receptors on the cell surface is the first step in the infection cycle. This HA-glycan interaction plays an important role in virus infection and transmission. This interaction has long been classified by the cis or trans conformation the glycan takes. Defining the binding specificity of HA based on the terminal linkage motif (Neu5Ac $\alpha 2-3 \mathrm{Gal}-$ or Neu5Ac $\alpha 2-3 \mathrm{Gal}-)$ alone provides a simplistic view of its complex multivalent interactions with a diverse set of glycans and does not readily correlate with human adaptation of HA and human-to-human transmission of these viruses. Capturing glycan characteristics beyond the terminal linkage has led to the classification of sialylated glycans taking conelike and umbrella-like topologies. The ability of HA to bind glycans in an umbrella-like topology appears critical for human adaptation and transmission. Most significantly, in defining distinct structural topologies for sialylated glycans, we put forth a concept to distinguish the $\alpha 2-3$ and $\alpha 2-6$ binding of avian HAs from that of the $\alpha 2-6$ binding of human adapted $\mathrm{H} 1$ and $\mathrm{H} 3 \mathrm{HAs}$ and enable the correlation of HA glycan specificity to human adaptation and transmission.

Acknowledgement This work was supported by the National Institute of General Medical Sciences glue grant U54 GM62116 and National Institutes of Health (NIH) grants GM57073 and the SingaporeMassachusetts Institute of Technology Alliance for Research and Technology (SMART). The authors would like to thank Ms. Ada Ziolkowski for help with the preparation of the manuscript. 


\section{References}

1. Varki, A., Cummings, R., Esko, J.D., Freeze, H., Hart, G.W., Marth, J.: Essentials of Glycobiology. Cold Spring Harber Laboratory Press, New York (1999)

2. Raman, R., Raguram, S., Venkataraman, G., Paulson, J.C., Sasisekharan, R.: Glycomics: an integrated systems approach to structure-function relationships of glycans. Nat. Methods 2, 817824 (2005). doi:10.1038/nmeth807

3. Sasisekharan, R., Raman, R., Prabhakar, V.: Glycomics approach to structure-function relationships of glycosaminoglycans. Annu. Rev. Biomed. Eng. 8, 181-231 (2006). doi:10.1146/annurev. bioeng.8.061505.095745

4. Bernfield, M., Gotte, M., Park, P.W., Reizes, O., Fitzgerald, M.L., Lincecum, J., et al: : Functions of cell surface heparan sulfate proteoglycans. Annu. Rev. Biochem. 68, 729-777 (1999). doi:10.1146/annurev.biochem.68.1.729

5. Suzuki, Y., Ito, T., Suzuki, T., Holland Jr., R.E., Chambers, T.M., Kiso, M., et al.: Sialic acid species as a determinant of the host range of influenza A viruses. J. Virol. 74, 11825-11831 (2000). doi:10.1128/JVI.74.24.11825-11831.2000

6. Skehel, J.J., Wiley, D.C.: Receptor binding and membrane fusion in virus entry: the influenza hemagglutinin. Annu. Rev. Biochem. 69, 531-569 (2000). doi:10.1146/annurev.biochem.69.1.531

7. Wiley, D.C., Skehel, J.J.: The structure and function of the hemagglutinin membrane glycoprotein of influenza virus. Annu. Rev. Biochem. 56, 365-394 (1987). doi:10.1146/annurev. bi.56.070187.002053

8. Stevens, J., Blixt, O., Paulson, J.C., Wilson, I.A.: Glycan microarray technologies: tools to survey host specificity of influenza viruses. Nat. Rev. Microbiol. 4, 857-864 (2006). doi:10.1038/nrmicro1530

9. Steinhauer, D.A., Skehel, J.J.: Genetics of influenza viruses. Annu. Rev. Genet. 36, 305-332 (2002). doi:10.1146/annurev. genet.36.052402.152757

10. Basler, C., Palese, P.: Influenza viruses. In: Creighton, T. (ed.) Encyclopedia of Molecular Medicine, pp. 1741-1747. Wiley, New York (2002)

11. Palese, P.: Influenza: old and new threats. Nat. Med. 10, S82-S87 (2004). doi:10.1038/nm1141

12. Tumpey, T.M., Garcia-Sastre, A., Taubenberger, J.K., Palese, P., Swayne, D.E., Basler, C.F.: Pathogenicity and immunogenicity of influenza viruses with genes from the 1918 pandemic virus. Proc. Natl Acad. Sci. USA 101, 3166-3171 (2004). doi:10.1073/ pnas. 0308391100

13. Chen, R.L., Lander, A.D.: Mechanisms underlying preferential assembly of heparan sulfate on glypican-1. J. Biol. Chem. 276, 7507-7517 (2001). doi:10.1074/jbc.M008283200

14. Nelson, M.I., Holmes, E.C.: The evolution of epidemic influenza. Nat. Rev. Genet. 8, 196-205 (2007). doi:10.1038/nrg2053

15. Kuiken, T., Holmes, E.C., McCauley, J., Rimmelzwaan, G.F., Williams, C.S., Grenfell, B.T.: Host species barriers to influenza virus infections. Science 312, 394-397 (2006). doi:10.1126/ science. 1122818

16. Matrosovich, M., Tuzikov, A., Bovin, N., Gambaryan, A., Klimov, A., Castrucci, M.R., et al.: Early alterations of the receptor-binding properties of $\mathrm{H} 1, \mathrm{H} 2$, and $\mathrm{H} 3$ avian influenza virus hemagglutinins after their introduction into mammals. J. Virol. 7(4), 8502-8512 (2000). doi:10.1128/JVI.74.18.8502-8512.2000

17. Neumann, G., Kawaoka, Y.: Host range restriction and pathogenicity in the context of influenza pandemic. Emerg. Infect. Dis. 12, 881-886 (2006)

18. Nguyen, D.C., Uyeki, T.M., Jadhao, S., Maines, T., Shaw, M., Matsuoka, Y., et al.: Isolation and characterization of avian influenza viruses, including highly pathogenic $\mathrm{H} 5 \mathrm{~N} 1$, from poultry in live bird markets in Hanoi, Vietnam, in 2001. J. Virol. 79, 4201-4212 (2005). doi:10.1128/JVI.79.7.4201-4212.2005
19. Perrone, L.A., Tumpey, T.M.: Reconstruction of the 1918 pandemic influenza virus: how revealing the molecular secrets of the virus responsible for the worst pandemic in recorded history can guide our response to future influenza pandemics. Infect. Disord. Drug Targets 7, 294-303 (2007). doi:10.2174/187152607783018772

20. Taubenberger, J.K., Reid, A.H., Lourens, R.M., Wang, R., Jin, G., Fanning, T.G.: Characterization of the 1918 influenza virus polymerase genes. Nature 437, 889-893 (2005). doi:10.1038/nature04230

21. Lee, C.W., Suarez, D.L., Tumpey, T.M., Sung, H.W., Kwon, Y.K., Lee, Y.J., et al.: Characterization of highly pathogenic H5N1 avian influenza A viruses isolated from South Korea. J. Virol. 79, 3692-3702 (2005). doi:10.1128/JVI.79.6.3692-3702.2005

22. Maines, T.R., Lu, X.H., Erb, S.M., Edwards, L., Guarner, J., Greer, P. W., et al.: Avian influenza (H5N1) viruses isolated from humans in Asia in 2004 exhibit increased virulence in mammals. J. Virol. 79, 11788-11800 (2005). doi:10.1128/JVI.79.18.11788-11800.2005

23. Tran, T.H., Nguyen, T.L., Nguyen, T.D., Luong, T.S., Pham, P.M., Nguyen, V.C., et al.: Avian influenza A (H5N1) in 10 patients in Vietnam. N Engl J. Med. 350, 1179-1188 (2004). doi:10.1056/ NEJMoa040419

24. Tumpey, T.M., Suarez, D.L., Perkins, L.E., Senne, D.A., Lee, J., Lee, Y.J., et al: : Evaluation of a high-pathogenicity H5N1 avian influenza A virus isolated from duck meat. Avian Dis. 47, 951-955 (2003)

25. de Jong, M.D., Simmons, C.P., Thanh, T.T., Hien, V.M., Smith, G. J., Chau, T.N., et al.: Fatal outcome of human influenza A (H5N1) is associated with high viral load and hypercytokinemia. Nat. Med. 12, 1203-1207 (2006). doi:10.1038/nm1477

26. Maines, T.R., Chen, L.M., Matsuoka, Y., Chen, H., Rowe, T., Ortin, J., et al.: Lack of transmission of H5N1 avian-human reassortant influenza viruses in a ferret model. Proc. Natl Acad. Sci. USA 103, 12121-12126 (2006). doi:10.1073/pnas.0605134103

27. Yen, H.L., Lipatov, A.S., Ilyushina, N.A., Govorkova, E.A., Franks, J., Yilmaz, N., et al.: Inefficient transmission of $\mathrm{H} 5 \mathrm{~N} 1$ influenza viruses in a ferret contact model. J. Virol. 81, 6890 6898 (2007). doi:10.1128/JVI.00170-07

28. Wilson, I.A., Skehel, J.J., Wiley, D.C.: Structure of the haemagglutinin membrane glycoprotein of influenza virus at $3 \mathrm{~A}$ resolution. Nature 289, 366-373 (1981). doi:10.1038/289366a0

29. Hirst, G.K.: The agglutination of red cells by allantoic fluid of chick embryos infected with influenza virus. Science 94, 22-23 (1941). doi:10.1126/science.94.2427.22

30. Gottschalk, A., Lind, P.E.: Product of interaction between influenza virus enzyme and ovomucin. Nature 164, 232 (1949). doi: $10.1038 / 164232 \mathrm{a} 0$

31. Klenk, E., Faillard, H., Lempfrid, H.: Enzymatic effect of the influenza virus. Hoppe Seylers Z. Physiol. Chem. 301, 235-246 (1955)

32. Bohm, P., Ross, J., Baumeister, L.: Cleavage of N-acetylneuraminic acid from serum by the receptor-destroying enzyme from Vibrio cholerae. Hoppe Seylers Z. Physiol. Chem. 307, 284-285 (1957)

33. Zilliken, F., Werner, G.H., Silver, R.K., Gyorgy, P.: Studies on the enzymatic properties of influenza viruses. I. The action of influenza B virus and RDE on the hemagglutinin inhibitor of human meconium. Virology 3, 464-474 (1957). doi:10.1016/ 0042-6822(57)90003-X

34. Traving, C., Schauer, R.: Structure, function and metabolism of sialic acids. Cell. Mol. Life Sci. 54, 1330-1349 (1998). doi:10.1007/ s000180050258

35. Varki, N.M., Varki, A.: Diversity in cell surface sialic acid presentations: implications for biology and disease. Lab. Invest. 87, 851-857 (2007). doi:10.1038/labinvest.3700656

36. Baum, L.G., Paulson, J.C.: Sialyloligosaccharides of the respiratory epithelium in the selection of human influenza virus receptor specificity. Acta Histochem. Suppl. 40, 35-38 (1990)

37. Shinya, K., Ebina, M., Yamada, S., Ono, M., Kasai, N., Kawaoka, Y.: Avian flu: influenza virus receptors in the human airway. Nature 440, 435-436 (2006). doi:10.1038/440435a 
38. Ito, T., Couceiro, J.N., Kelm, S., Baum, L.G., Krauss, S., Castrucci, M.R., et al:: Molecular basis for the generation in pigs of influenza A viruses with pandemic potential. J. Virol. 72, 7367-7373 (1998)

39. Eisen, M.B., Sabesan, S., Skehel, J.J., Wiley, D.C.: Binding of the influenza A virus to cell-surface receptors: structures of five hemagglutinin-sialyloligosaccharide complexes determined by Xray crystallography. Virology 232, 19-31 (1997). doi:10.1006/ viro.1997.8526

40. Ha, Y., Stevens, D.J., Skehel, J.J., Wiley, D.C.: X-ray structures of $\mathrm{H} 5$ avian and $\mathrm{H} 9$ swine influenza virus hemagglutinins bound to avian and human receptor analogs. Proc. Natl Acad. Sci. USA 98, 11181-11186 (2001). doi:10.1073/pnas.201401198

41. Ha, Y., Stevens, D.J., Skehel, J.J., Wiley, D.C.: X-ray structure of the hemagglutinin of a potential H3 avian progenitor of the 1968 Hong Kong pandemic influenza virus. Virology 309, 209-218 (2003). doi:10.1016/S0042-6822(03)00068-0

42. Russell, R.J., Gamblin, S.J., Haire, L.F., Stevens, D.J., Xiao, B., $\mathrm{Ha}$, Y., et al:: $\mathrm{H} 1$ and $\mathrm{H} 7$ influenza haemagglutinin structures extend a structural classification of haemagglutinin subtypes. Virology 325, 287-296 (2004). doi:10.1016/j.virol.2004.04.040

43. Russell, R.J., Stevens, D.J., Haire, L.F., Gamblin, S.J., Skehel, J. J.: Avian and human receptor binding by hemagglutinins of influenza A viruses. Glycoconj. J. 23, 85-92 (2006). doi:10.1007/ s10719-006-5440-1

44. Stevens, J., Blixt, O., Tumpey, T.M., Taubenberger, J.K., Paulson, J. C., Wilson, I.A.: Structure and receptor specificity of the hemagglutinin from an H5N1 influenza virus. Science 312, 404-410 (2006). doi:10.1126/science.1124513

45. Yamada, S., Suzuki, Y., Suzuki, T., Le, M.Q., Nidom, C.A., SakaiTagawa, Y., et al.: Haemagglutinin mutations responsible for the binding of $\mathrm{H} 5 \mathrm{~N} 1$ influenza A viruses to human-type receptors. Nature 444, 378-382 (2006). doi:10.1038/nature05264

46. Gamblin, S.J., Haire, L.F., Russell, R.J., Stevens, D.J., Xiao, B., Ha, Y., et al.: The structure and receptor binding properties of the 1918 influenza hemagglutinin. Science 303, 1838-1842 (2004). doi:10.1126/science. 1093155

47. Connor, R.J., Kawaoka, Y., Webster, R.G., Paulson, J.C.: Receptor specificity in human, avian, and equine $\mathrm{H} 2$ and $\mathrm{H} 3$ influenza virus isolates. Virology 205, 17-23 (1994). doi:10.1006/viro.1994.1615

48. Rogers, G.N., Paulson, J.C.: Receptor determinants of human and animal influenza virus isolates: differences in receptor specificity of the $\mathrm{H} 3$ hemagglutinin based on species of origin. Virology 127, 361-373 (1983). doi:10.1016/0042-6822(83)90150-2

49. Paulson, J.C., Rogers, G.N.: Resialylated erythrocytes for assessment of the specificity of sialyloligosaccharide binding proteins. Methods Enzymol 138, 162-168 (1987). doi:10.1016/0076-6879(87)38013-9

50. Tumpey, T.M., Maines, T.R., Van Hoeven, N., Glaser, L., Solorzano, A., Pappas, C., et al.: A two-amino acid change in the hemagglutinin of the 1918 influenza virus abolishes transmission. Science 315, 655-659 (2007). doi:10.1126/science.1136212

51. Yang, Z.Y., Wei, C.J., Kong, W.P., Wu, L., Xu, L., Smith, D.F., et al.: Immunization by avian h5 influenza hemagglutinin mutants with altered receptor binding specificity. Science 317, 825-828 (2007). doi:10.1126/science.1135165

52. Gambaryan, A.S., Tuzikov, A.B., Pazynina, G.V., Desheva, J.A., Bovin, N.V., Matrosovich, M.N., et al.: 6-sulfo sialyl Lewis X is the common receptor determinant recognized by $\mathrm{H} 5, \mathrm{H} 6, \mathrm{H} 7$ and H9 influenza viruses of terrestrial poultry. Virol. J. 5, 85 (2008). doi:10.1186/1743-422X-5-85

53. Gambaryan, A., Yamnikova, S., Lvov, D., Tuzikov, A., Chinarev, A., Pazynina, G., et al.: Receptor specificity of influenza viruses from birds and mammals: new data on involvement of the inner fragments of the carbohydrate chain. Virology 334, 276-283 (2005). doi:10.1016/j.virol.2005.02.003

54. Gambaryan, A.S., Piskarev, V.E., Yamskov, I.A., Sakharov, A.M., Tuzikov, A.B., Bovin, N.V., et al.: Human influenza virus recognition of sialyloligosaccharides. FEBS Lett. 366, 57-60 (1995). doi:10.1016/0014-5793(95)00488-U

55. Gambaryan, A.S., Matrosovich, M.N.: A solid-phase enzymelinked assay for influenza virus receptor-binding activity. J. Virol. Methods 39, 111-123 (1992). doi:10.1016/0166-0934(92)90130-6

56. Hanson, S., Best, M., Bryan, M.C., Wong, C.H.: Chemoenzymatic synthesis of oligosaccharides and glycoproteins. Trends Biochem. Sci. 29, 656-663 (2004). doi:10.1016/j.tibs.2004.10.004

57. Blixt, O., Razi, N.: Chemoenzymatic synthesis of glycan libraries. Methods Enzymol. 415, 137-153 (2006). doi:10.1016/S00766879(06)15009-0

58. Seeberger, P.H., Werz, D.B.: Synthesis and medical applications of oligosaccharides. Nature 446, 1046-1051 (2007). doi:10.1038/ nature 05819

59. Blixt, O., Head, S., Mondala, T., Scanlan, C., Huflejt, M.E., Alvarez, R., et al.: Printed covalent glycan array for ligand profiling of diverse glycan binding proteins. Proc. Natl Acad. Sci. USA 101, 17033-17038 (2004). doi:10.1073/pnas.0407902101

60. Song, X., Xia, B., Lasanajak, Y., Smith, D.F., Cummings, R.D.: Quantifiable fluorescent glycan microarrays. Glycoconj. J. 25, 1525 (2008). doi:10.1007/s10719-007-9066-8

61. Kumari, K., Gulati, S., Smith, D.F., Gulati, U., Cummings, R.D., Air, G.M.: Receptor binding specificity of recent human H3N2 influenza viruses. Virol. J. 4, 42 (2007). doi:10.1186/1743-422X-4-42

62. Gambaryan, A.S., Tuzikov, A.B., Piskarev, V.E., Yamnikova, S.S., Lvov, D.K., Robertson, J.S., et al.: Specification of receptorbinding phenotypes of influenza virus isolates from different hosts using synthetic sialylglycopolymers: non-egg-adapted human H1 and $\mathrm{H} 3$ influenza $\mathrm{A}$ and influenza $\mathrm{B}$ viruses share a common high binding affinity for 6 '-sialyl(N-acetyllactosamine). Virology $\mathbf{2 3 2}$, 345-350 (1997). doi:10.1006/viro.1997.8572

63. Tumpey, T.M., Basler, C.F., Aguilar, P.V., Zeng, H., Solorzano, A., Swayne, D.E., et al.: Characterization of the reconstructed 1918 Spanish influenza pandemic virus. Science 310, 77-80 (2005). doi:10.1126/science.1119392

64. Matrosovich, M., Matrosovich, T., Carr, J., Roberts, N.A., Klenk, H.D.: Overexpression of the alpha-2, 6-sialyltransferase in MDCK cells increases influenza virus sensitivity to neuraminidase inhibitors. J. Virol. 77, 8418-8425 (2003). doi:10.1128/JVI.77.15.8418-8425.2003

65. Matrosovich, M., Zhou, N., Kawaoka, Y., Webster, R.: The surface glycoproteins of $\mathrm{H} 5$ influenza viruses isolated from humans, chickens, and wild aquatic birds have distinguishable properties. J. Virol. 73, 1146-1155 (1999)

66. Raman, R., Sasisekharan, R.: Cooperativity in glycan-protein interactions. Chem. Biol. 14, 873-874 (2007). doi:10.1016/j. chembiol.2007.08.003

67. Chandrasekaran, A., Srinivasan, A., Raman, R., Viswanathan, K., Raguram, S., Tumpey, T.M., et al.: Glycan topology determines human adaptation of avian $\mathrm{H} 5 \mathrm{~N} 1$ virus hemagglutinin. Nat. Biotechnol. 26, 107-113 (2008). doi:10.1038/nbt1375

68. Nicholls, J.M., Bourne, A.J., Chen, H., Guan, Y., Peiris, J.S.: Sialic acid receptor detection in the human respiratory tract: evidence for widespread distribution of potential binding sites for human and avian influenza viruses. Respir. Res. 8, 73 (2007). doi:10.1186/1465-9921-8-73

69. Bewley, C.A.: Illuminating the switch in influenza viruses. Nat. Biotechnol. 26, 60-62 (2008). doi:10.1038/nbt0108-60

70. Stevens, J., Blixt, O., Chen, L.M., Donis, R.O., Paulson, J., Wilson, I.A.: Recent avian H5N1 viruses exhibit increased propensity for acquiring human receptor specificity. J. Mol. Biol. 381, (5):1382-94 (2008). doi:10.1016/j.jmb.2008.04.016

71. Srinivasan, A., Viswanathan, K., Raman, R., Chandrasekaran, A., Raguram, S., Tumpey, T.M., et al.: Quantitative biochemical rationale for differences in transmissibility of 1918 pandemic influenza A viruses. Proc. Natl Acad. Sci. USA 105, 2800-2805 (2008). doi:10.1073/pnas.0711963105 